\title{
Inhibition of neuroblastoma cell proliferation with omega-3 fatty acids and treatment of a murine model of human neuroblastoma using a diet enriched with omega-3 fatty acids in combination with sunitinib
}

Carmen M. Barnés', Daniela Prox', Emily A. Christison-Lagay², Hau D. Le², Sarah Short', Flavia Cassiola', Dipak Panigrahy', Deviney Chaponis', Catherine Butterfield', Deepika Nehra², Erica M. Fallon², Mark Kieran², Judah Folkman ${ }^{1,4}$ and Mark Puder ${ }^{2}$

INTRODUCTION: We investigated the use of dietary omega-3 $(\omega-3)$ polyunsaturated fatty acids (PUFAs) in the treatment of neuroblastoma both as a sole agent and in combination with sunitinib, a broad-spectrum tyrosine kinase receptor inhibitor. RESULTS: Substitution of all dietary fat with menhaden oil ( $\omega-3$ PUFA rich) resulted in a $40-70 \%$ inhibition of tumor growth and a statistically significant difference in the levels of several PUFAs (18:2 $\omega-6,20: 4 \omega-6,22: 4 \omega-6,20: 5 \omega-3)$ as compared with a control diet. Furthermore, tumors from animals on the $\omega-3$ fatty acid (FA)-enriched diet had an elevated triene/tetraene ratio suggestive of a change in local eicosanoid metabolism in these tissues similar to that seen with essential fatty acid deficiency. The $\omega-3$ FA-enriched diet also decreased tumor-associated inflammatory cells and induced mitochondrial changes suggestive of mitochondrial damage. Combination treatment with sunitinib resulted in further reduction in tumor proliferation and microvessel density. DISCUSSION: These findings suggest a potential role for $\omega-3$ PUFAs in the combination treatment of neuroblastoma.

METHODS: We used a murine model of orthotopic and subcutaneous human neuroblastoma and diets that differ in the FA content to define the optimal dietary $\omega-3 /$ omega- 6 ( $\omega-6)$ FA ratio required for the inhibition of these tumors.

N euroblastoma is the most common extracranial solid organ tumor of infancy (1). It accounts for $7-8 \%$ of all childhood cancers and nearly $15 \%$ of pediatric oncology deaths, making it the most deadly extracranial malignancy of childhood.

Fatty acids (FAs), originally thought to be purely an energy source, have proven to be highly active molecules that play major roles in the regulation of metabolic pathways and inflammatory responses. The omega- $6(\omega-6) \mathrm{FA}$, arachidonic acid (AA; 20:4 $\omega-6)$, and omega-3 ( $\omega-3)$ FAs, eicosapentaenoic acid (EPA; 20:5 $\omega-3$ ) and docosahexaenoic acid (DHA; 22:6 $\omega-3$ ), are integral components of the cell membrane and are highly active molecules in the FA metabolic pathway. AA derives from linoleic acid (LA; 18:2 $\omega-6$ ), whereas EPA and DHA derive from $a$-linolenic acid (18:3 $\omega-3)$. In humans, the latter conversion is poor, making direct ingestion of EPA and DHA the best method of increasing long-chain $\omega-3$ FA content. AA, EPA, and DHA are metabolized to eicosanoids, biologically active lipids that modulate cell growth, inflammation, immunity, platelet aggregation, and angiogenesis. AA metabolites are generally proinflammatory, prothrombotic, and vasoconstricting, whereas EPA and DHA derivatives are anti-inflammatory and vasodilating.

Western diets contain disproportionally high $\omega-6$ and low $\omega-3$ FAs, resulting in a high $\omega-6 / \omega-3$ ratio that has been linked to multiple pathological conditions. Animal and human studies suggest that decreasing the $\omega-6 / \omega-3$ ratio ameliorates cardiovascular disease and improves other outcomes (2). Recently, there has been a growing interest in exploring the role of $\omega-3$ FAs in several disease conditions (3). Although the efficacy of $\omega-3$ FAs in human cancer remains inconclusive, in vitro and in vivo animal studies suggest that $\omega-3$ FAs may have a protective effect against breast, prostate, liver, colon, and skin cancer in addition to neuroblastoma (4-8).

In this study, we investigated the effect of an $\omega-3$ FA-enriched diet on neuroblastoma tumor growth and attempt to define an optimum dietary ratio of $\omega-6 / \omega-3$ FAs in an orthotopic and subcutaneous murine xenograft tumor model. As vascular endothelial growth factor expression and a vascular phenotype correlate with metastasis and poor clinical outcome in neuroblastoma (9), we also evaluated the effect of the FDA-approved agent sunitinib (Sutent; SU11248; Pfizer, New York, NY), a vascular endothelial growth factor receptor, platelet-derived growth factor receptor, and kit inhibitor, in combination with an $\omega-3$ FA-enriched diet.

\section{RESULTS}

$\omega-3$ FAs and Sunitinib Have Direct Antitumor Effects on Neuroblastoma Cells In Vitro

DHA and AA resulted in a dose-dependent decrease in tumor cell proliferation. Human neuroblastoma (SK-NSH) cells were

\footnotetext{
'Vascular Biology Program, Children's Hospital Boston, Harvard Medical School, Boston, Massachusetts; ${ }^{2}$ Department of Surgery, Children's Hospital Boston, Harvard Medical School, Boston, Massachusetts; ${ }^{3}$ Department of Pediatric Oncology, Dana Farber Cancer Institute; Harvard Medical School, Boston, Massachusetts; ${ }^{4}$ Deceased. Correspondence: Mark Puder (mark.puder@childrens.harvard.edu) 


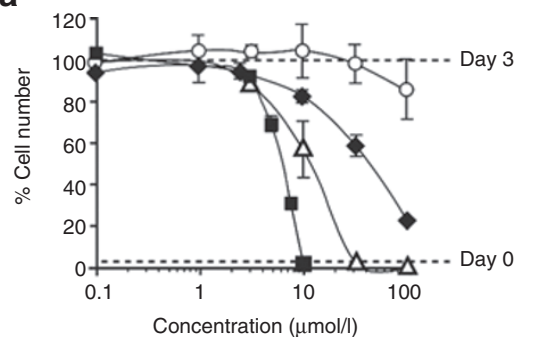

d

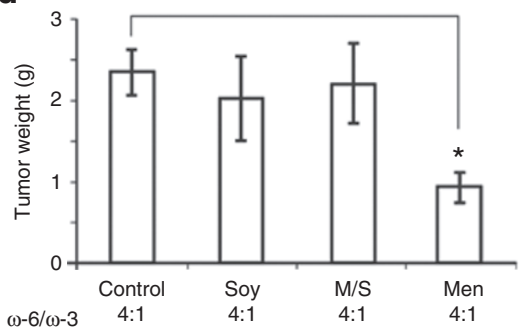

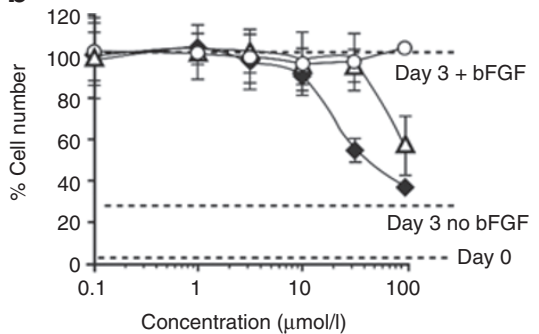

e

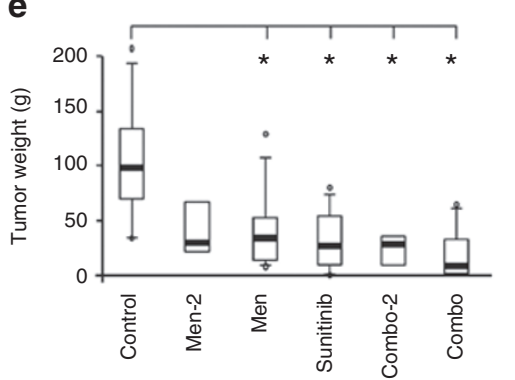

C
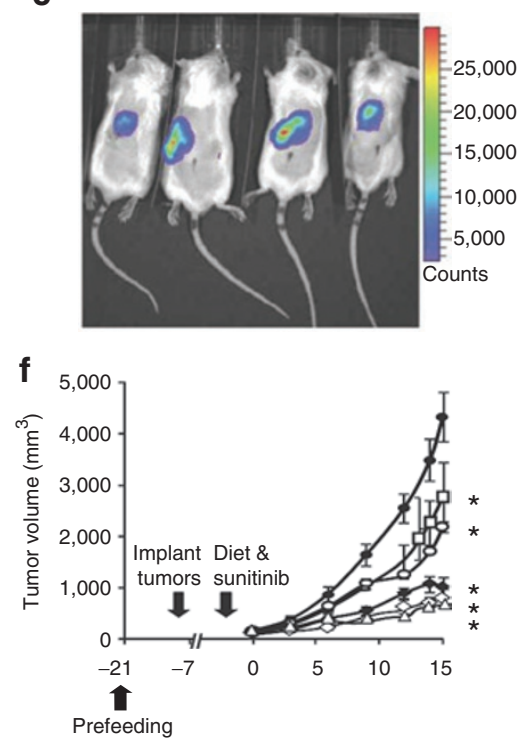

Figure 1. Inhibition of neuroblastoma cells and tumors. (a, b) In vitro: Survival curves of (a) neuroblastoma (SK-NSH) and (b) BCE cells, treated with DHA (open triangle), AA (filled diamond), HCO (open circle), or sunitinib (filled square), three independent experiments in triplicate. (c-f) In vivo: (c) Orthotopic control tumors visualized by luciferase imaging. (d) Tumor weight of orthotopic tumors in pretreatment group ( $n=5 /$ group) and (e) in treatment/ sunitinib groups ( $n=5-10 /$ group). (f) Subcutaneous neuroblastoma treated with Control (filled circle), Sunitinib (filled diamond), Men (open circle), Men-2 (open square), Combo (open diamond), or Combo-2 (open triangle) ( $n=8-14 /$ group). Tumor weights/volumes reported as median (interquartile range). ${ }^{*} P<0.001$. AA, arachidonic acid; BCE, bovine capillary endothelial; bFGF, basic fibroblast growth factor; Combo, Men + Sunitinib combination pretreatment group; Combo-2, combination treatment group (Men-2 + Sunitinib); DHA, docosahexaenoic acid; HCO, hydrogenated coconut oil; Men, group fed $10 \%$ wt/wt menhaden oil; Men-2, Men diet administered to mice with established tumors.

more sensitive to DHA than bovine capillary endothelial cells as measured by the half maximal inhibitory concentration $\left(\mathrm{IC}_{50}\right)(13.5 \mu \mathrm{mol} / \mathrm{l} \mathrm{vs} .>100 \mu \mathrm{mol} / \mathrm{l}$, respectively), whereas the response to AA was the same $\left(\mathrm{IC}_{50}=40 \mu \mathrm{mol} / \mathrm{l}\right)$. Neither cell type was affected by hydrogenated coconut oil (Figure 1a,b). Sunitinib was the most toxic to SK-NSH cells in vitro $\left(\mathrm{IC}_{50}=\right.$ $6.5 \mu \mathrm{mol} / \mathrm{l}$ ) (Figure 1a). The IMR-32 neuroblastoma cell line also exhibited a dose-dependent decrease in tumor cell proliferation with $80 \%$ of the baseline cell count at a DHA concentration of $100 \mu \mathrm{mol} / \mathrm{l}$.

Diets Enriched With $\omega-3$ FAs Inhibit Neuroblastoma Tumor Growth In Vivo

Animals were fed diets varying in $\omega-6 / \omega-3$ FA ratio $(8: 1-1: 10)$ and $\omega-3$ FA content $(0.51-3.3 \%)$ (Table 1$)$ in addition to the type of $\omega-3$ FA provided. $\alpha$-Linolenic acid was the only $\omega-3$ polyunsaturated fatty acid (PUFA) in the Soy group, whereas long-chain PUFAs constituted 42,79 , and $85 \%$ of the total $\omega-3$ PUFA content in the Control, M/S (menhaden oil/soybean oil), and Men groups, respectively.

Before tumor implantation, animals were prefed for 3 weeks with the Control, Soy, M/S, or Men diet. Luciferase imaging was used to confirm orthotopic tumor take (Figure 1c); as photon flux did not correlate to tumor size, final tumor weights were necessary to assess efficacy. Only the Men diet decreased tumor volume in the orthotopic (60\%; Figure 1d,e) and subcutaneous models (40-60\%; Figure 1f). The Men diet administered to established tumors (Men-2) was almost as effective as when given prior to tumor implantation (Men) (Figure 1e,f).

\section{Men Diet and Sunitinib Have Comparable Inhibition of Neuroblastoma Tumor Growth In Vivo}

As single therapy, sunitinib $(20 \mathrm{mg} / \mathrm{kg} /$ day $)$ produced a $>60 \%$ inhibition of tumor growth (Figure 1e,f). In orthotopic tumors, the median values among all treatment groups differed significantly from the Control group, but not from one another (Figure 1e). Subcutaneous tumors in the Men, Sunitinib, and Men + Sunitinib combination group (Combo) were statistically smaller than control tumors $(P<0.001)$ (Figure 1f), with the Combo also differing from Sunitinib and Men $(P=0.003)$.

\section{Histological Characteristics of Neuroblastoma Tumors}

Irrespective of treatment, all tumors were "poorly differentiated" and necrotic according to the Pediatric Oncology Group classification. Non-necrotic regions from the outer rim of tumors were isolated for histology. Representative hematoxylin and eosin stains from orthotopic tumors are shown for the Control, Men, Sunitinib, and Combo groups (Figure 2a).

Although it has been proposed that $\omega-3$ FAs may reduce tumor growth by decreasing microvessel density (MVD), we found that the Men diet had no effect on tumor MVD. Only Sunitinib orthotopic (Figure 2c) and Combo subcutaneous tumors (data not shown) had a lower MVD than control tumors $(P<0.05)$.

Tumors were stained with the pan-hematopoietic marker CD45 to quantify the presence of tumor-associated monocytes, which are known to be proangiogenic and protumorigenic. Non-necrotic areas stained weakly for stromal-associated 
Table 1. Comparison of the diets used in the study

\begin{tabular}{|c|c|c|c|c|}
\hline & Control & Soy & $\mathrm{M} / \mathrm{S}$ & Men \\
\hline \multicolumn{5}{|l|}{ Composition (per $100 \mathrm{~g}$ diet) } \\
\hline Protein, $\mathrm{g}$ & 22.5 & 21.0 & 21.0 & 21.0 \\
\hline Carbohydrate, $\mathrm{g}$ & 50.1 & 45.2 & 45.2 & 45.2 \\
\hline Fat, g & 5.9 & 10 & 10 & 10 \\
\hline Fiber (crude, g) & 4.8 & 4.71 & 4.71 & 4.71 \\
\hline $\begin{array}{l}\text { Total digestible } \\
\text { nutrients, } \mathrm{g}\end{array}$ & 79.4 & 76.2 & 76.2 & 76.2 \\
\hline \multicolumn{5}{|l|}{ Calories provided by } \\
\hline Protein, \% & 26.2 & 19.1 & 19.1 & 19.1 \\
\hline Fat (ether extract), \% & 15.5 & 23.0 & 23.0 & 23.0 \\
\hline Carbohydrates, $\%$ & 58.3 & 55.8 & 55.8 & 55.7 \\
\hline \multicolumn{5}{|l|}{ Fat consumed (per $100 \mathrm{~g}$ diet) } \\
\hline Saturated FA (g) & 1.24 & 1.41 & 2.54 & 2.92 \\
\hline Unsaturated FA (g) & 4.59 & 7.60 & 6.55 & 6.20 \\
\hline Monounsaturated FA (g) & 1.27 & 2.09 & 2.44 & 2.56 \\
\hline$\omega-9 F A(g)$ & 1.07 & 2.21 & 1.47 & 1.22 \\
\hline PUFA (g) & 3.28 & 5.51 & 4.27 & 3.85 \\
\hline$\omega-3$ PUFA (g) & 0.57 & 0.64 & 2.64 & 3.31 \\
\hline 18:3 (n-3) ALA (g) & 0.30 & 0.64 & 0.23 & 0.10 \\
\hline 20:5 (n-3) EPA (g) & 0.09 & 0.00 & 1.09 & 1.45 \\
\hline 22:5 (n-3) DPA (g) & 0.02 & 0.00 & 0.17 & 0.23 \\
\hline 22:6 (n-3) DHA (g) & 0.12 & 0.00 & 0.78 & 1.04 \\
\hline$\omega-6$ PUFA (g) & 2.69 & 5.13 & 1.53 & 0.33 \\
\hline $20: 4(n-6) A A(g)$ & 0.00 & 0.00 & 0.06 & 0.09 \\
\hline $18: 2(n-6)$ LA (g) & 2.67 & 5.13 & 1.38 & 0.14 \\
\hline Unsaturated/saturated & 3.70 & 5.39 & 2.58 & 2.12 \\
\hline $\begin{array}{l}\text { Polyunsaturated/ } \\
\text { saturated }\end{array}$ & 2.73 & 3.91 & 1.68 & 1.32 \\
\hline$\omega-6 / \omega-3$ & 4.76 & 8.02 & 0.58 & 0.10 \\
\hline $\begin{array}{l}\text { Daily food intake } \\
\text { (g/kg/day) }\end{array}$ & $130 \pm 20$ & $104 \pm 13$ & $120 \pm 20$ & $93 \pm 19$ \\
\hline Daily fat intake (g/kg/day) & $7.7 \pm 1.2$ & $10 \pm 1.3$ & $12 \pm 20$ & $9.3 \pm 1.9$ \\
\hline
\end{tabular}

AA, arachidonic acid; ALA, a-linolenic acid; DHA, docosahexaenoic acid; DPA, docosapentaenoic acid; EPA, eicosapentaenoic acid; FA, fatty acid; LA, linoleic acid; M/S, $7.5 \%$ wt/wt menhaden oil + 2.5\% wt/wt soybean oil; Men, $10 \%$ wt/wt menhaden oil; PUFA, polyunsaturated fatty acid; Soy, $10 \% \mathrm{wt} / \mathrm{wt}$ soybean oil.

CD $45^{+}$cells, which were twice as prevalent in the subcutaneous as compared with the orthotopic control tumors. CD $45^{+}$ stromal cells were nearly absent in Men, Sunitinib, and Combo subcutaneous tumors $(P=0.001$, data not shown) and in Sunitinib orthotopic tumors ( $0.6 \%$ of control tumors) (Figure 2d,e). Men orthotopic tumors had $\sim 80 \%$ fewer CD $45^{+}$ cells than Soy, M/S, and Control tumors (Figure 2e).

TUNEL and Ki67 staining was performed to determine rates of apoptosis and proliferation, respectively, in the neuroblastoma tumor xenografts. The proliferation index was significantly decreased only in the Combo group (Figure 2f). Non-necrotic sections had few apoptotic nuclei (Figure $2 \mathrm{~g}$, left) and were not significantly different from controls. Only areas surrounding or within necrotic areas (Figure 2g, middle, right) had increased TUNEL staining.

\section{Ultrastructural Characteristics of Neuroblastoma Tumors}

All tumors were poorly differentiated, and tumor cells had an ovoid shape, sparse cytoplasm, short and sparse neuritic processes, and damaged mitochondria (Figure 3a). There was no increase in neurosecretory granules or microtubules, which hallmark cellular differentiation (10), in any treatment group (4-8\% of neuroblastoma cells had neurosecretory granules across all groups).

\section{Lipid Bodies}

Lipid bodies contain cyclooxygenase-2 (COX-2) and actively participate in lipid metabolism and inflammation via prostaglandin E2 synthesis (11). These lipid bodies are generally more prevalent in neoplastic cells and correlate with tumor growth (11). We found lipid bodies to be present in all tumors, with the highest accumulation in the Soy and Men groups (Figure 3b). The lipid composition in lipid bodies varied with treatment group, with electron-lucent droplets in the Soy and Control tumors and electron-dense vacuoles in the Men tumors (Figure $3 \mathrm{~b}$ ). The dark contrast, caused by the reaction of osmium tetraoxide with the double bonds of FAs, correlated only with EPA and DHA, rather than total FA content (Figure $3 \mathrm{c}$ and Table 1 ). This suggests that lipid body-derived eicosanoids in Men-fed animals are likely to be derived from DHA and EPA and may be anti-inflammatory, in contrast to those produced in Control and Soy-fed mice.

\section{FA Profiles in Control, Soy, M/S, and Men Tissues}

To investigate the FA variation within tumors, we extracted lipids from 75 tissue samples from tumor-bearing mice (29 tumors, 26 livers, 19 skeletal muscle). Hierarchical clustering was performed to determine similarities in lipid composition and to determine whether the lipid composition within a tissue was primarily determined by organ type or diet (Figure 4). Despite the dramatic differences in FA composition between the four diets, all samples grouped first by tissue type, with $95 \%$ of all samples (70/74) segregating correctly into a tumor branch or a muscle/liver branch. Within each tissue branch, the samples then sorted by diet into a Soy-Control or a Men-M/S cluster.

\section{Low LA, AA, and Adrenic Acid and High EPA in Tumors From Animals Fed the Men Diet}

As only the Men diet inhibited neuroblastoma tumor growth, we evaluated whether there were any differences in FA profiles between (i) Men vs. M/S tumors and (ii) Men vs. all other groups combined. When orthotopic and subcutaneous tumors were analyzed jointly, only four FAs differed between Men and M/S tumors and all groups combined (Tables 2 and 3; $P<0.05)$ : LA, AA, EPA, and adrenic acid (22:4 $\omega-6)$. LA is a precursor to AA, and AA is a precursor to inflammatory eicosanoids, whereas EPA is a precursor to anti-inflammatory eicosanoids. Men tumors also had lower total $\omega-6$ FA and PUFA content, and a lower $\omega-6 / \omega-3$ FA ratio. The above-mentioned 

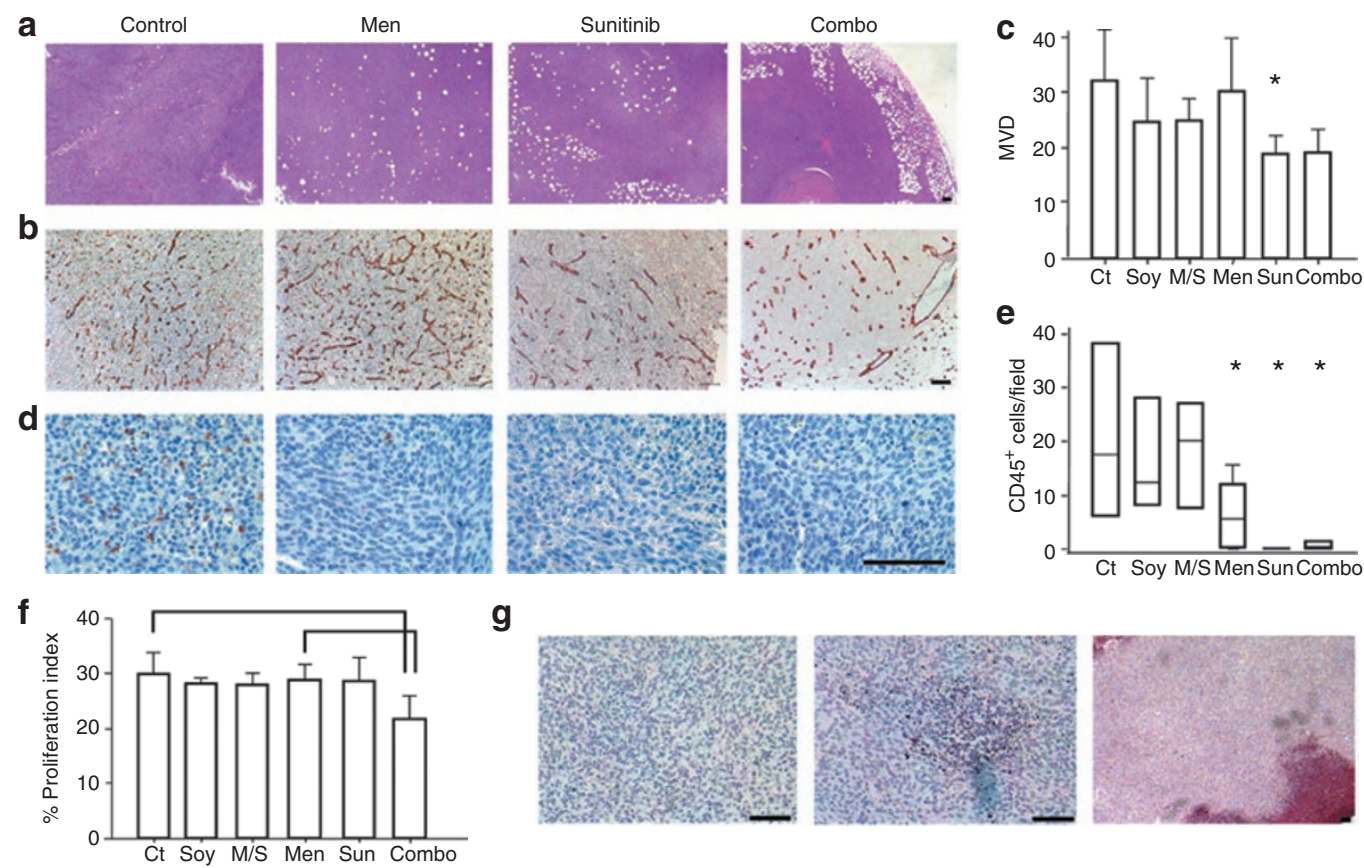

g
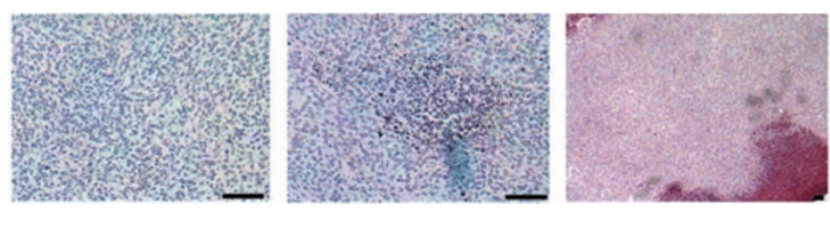

Figure 2. Immunohistochemistry of orthotopic tumors. (a) Representative hematoxylin and eosin ( $\mathrm{H}+\mathrm{E})$-stained orthotopic tumors (original magnification $\times 4$ ). MVD: (b) CD31 staining and (c) quantification (mean $\pm S D, n=4-7 /$ group). Tumor-associated inflammatory cells: (d) CD45 staining (in red, nuclei in blue, original magnification $\times 40$ ) and (e) quantification (median + interquartile range; 4-6/group). Proliferation index: (f) with Ki67/DAPI and apoptosis (g) with TUNEL staining with representative sections with no apoptotic cells (Control, left), region with apoptotic cells (Soy, middle), large areas of necrosis (Men, right). Scale bar is $100 \mu \mathrm{m}$ in all panels. ${ }^{*} P<0.05$. Ct, Control; Men, group fed $10 \%$ wt/wt menhaden oil; MVD, microvessel density; Soy, group fed $10 \%$ wt/wt soybean oil; Sun, Sunitinib group.

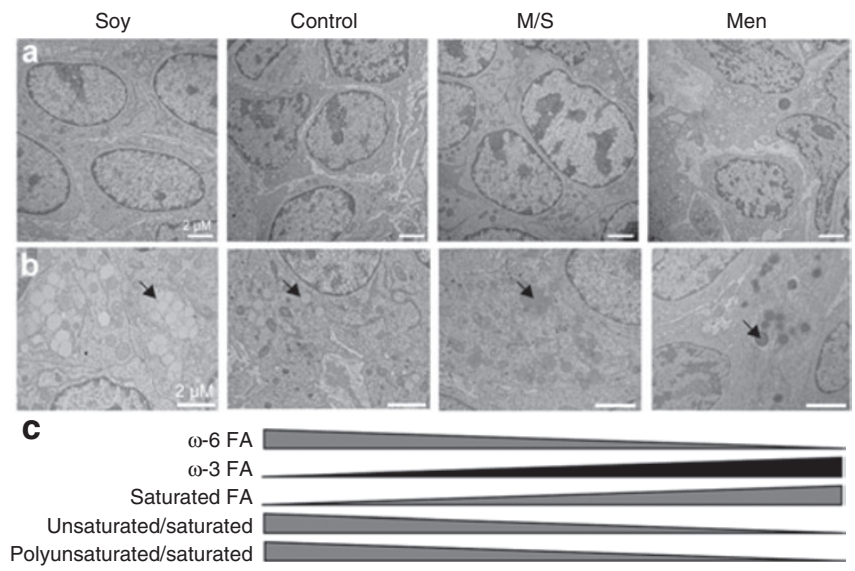

Figure 3. Lipid accumulation in neuroblastoma cells. (a) EM showing ultrastructural details of neuroblastoma tumors; scale bar $=2 \mu \mathrm{m}$. (b) Lipid accumulation was highest in the Soy and Men groups. (c) Darkness of the fat vacuoles correlated with increasing $\omega-3$ FA content and not with the unsaturated/saturated FA ratio. EM, electron microscopy; FA, fatty acid; Men, group fed 10\% wt/wt menhaden oil; Soy, group fed 10\% wt/wt soybean oil.

FAs (except adrenic acid) were also found to be different in livers (Tables 3 and 4). Men livers also had higher DHA and total $\omega-3$ FA content and a lower dihomo- $\gamma$-linolenic acid (20:3 $\omega-6)$ content, another precursor to less inflammatory eicosanoids. In addition, the 20:3 $\omega-6 / 18: 2 \omega-6$ (delta- 6 desaturase) and 20:4 $\omega-6 / 20: 3 \omega-6$ (delta-5 desaturase) ratios were increased in Men livers, suggesting an accelerated hepatic conversion of
LA to AA. Thus, although AA was significantly lower in Men tumors, Men livers could regulate their AA content, showing comparable levels to $\mathrm{M} / \mathrm{S}$-fed animals.

\section{Tumors but Not Tissues of Animals Fed the Men Diet Are Characterized by a High Triene:Tetraene Ratio}

A Mead acid (20:3 $\omega-9)$ :AA (triene:tetraene) ratio of $>0.2$ is suggestive of biochemical essential FA deficiency. All Men tumors had a significantly increased triene:tetraene ratio $(0.23$ and 0.36 , respectively). Subcutaneous but not orthotopic M/S tumors had a triene:tetraene ratio $>0.2(0.24)$. In contrast to tumors, the liver triene:tetraene ratios from tumor-bearing animals were low at $0.009 \pm 0.004$ in the Men and $0.011 \pm 0.002$ in the M/S group, and Mead acid was undetectable in muscle (Table 5).

\section{Decreased Metabolism of AA in Men Tumors}

Phospholipase A2 (PLA-2) is required for AA release from membrane phospholipids and its subsequent conversion to inflammatory metabolites by COX and lipoxygenases. A critical role in eicosanoic formation and tumorigenesis has been demonstrated for cytosolic PLA-2 $(12,13)$. We speculated that the low $\omega-6$ FAs in Men tumors would be less available for conversion by PLA-2 and COX-2. Western blot analysis for protein levels in tumor lysates demonstrated that total PLA-2 was significantly decreased in Men tumors relative to the those in the Control and Soy groups. COX-2, which is highly expressed in neuroblastoma tumor cells, was unaltered by diet (Figure 5). 


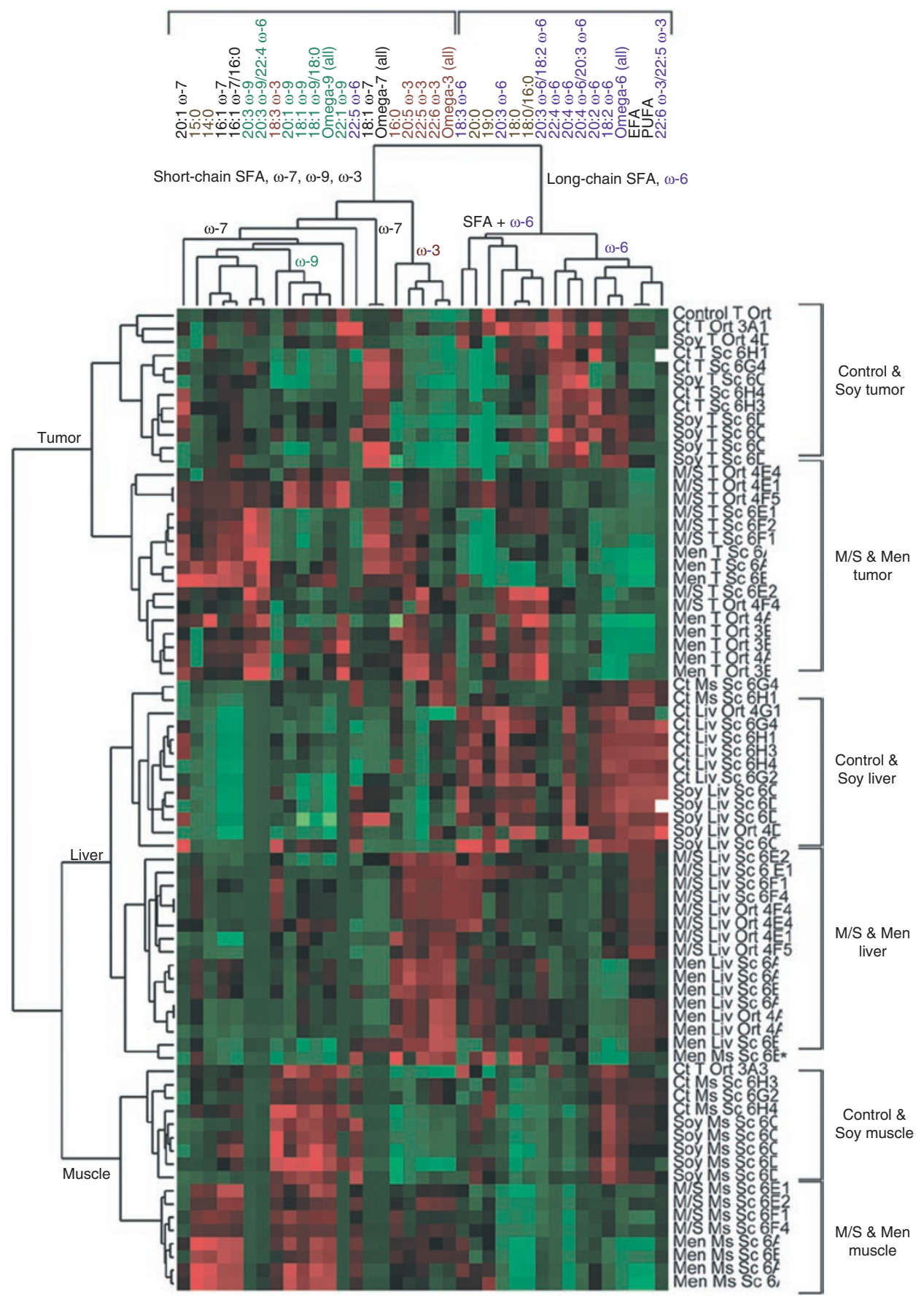

Figure 4. Heat map of hierarchical clustering of FAs. Spearman rank correlation used as the similarity metric and centroid linkage as clustering method. ${ }^{*} P<0.05$. Ct, Control; EFA, essential FA; FA, fatty acid; Liv, liver; Men, group fed 10\% wt/wt menhaden oil; Ms, muscle; M/S, group fed 7.5\% wt/wt menhaden oil $+2.5 \% \mathrm{wt} / \mathrm{wt}$ soybean oil; Ort, orthotopic tumor; Sc, subcutaneous tumor; SFA, saturated FA; T, tumor.

\section{DISCUSSION}

This is the first study to address the effect of the $\omega-3 / \omega-6$ FA ratio in the inhibition of neuroblastoma growth in vivo and to investigate the mechanism of action in subcutaneous and orthotopic tumor models. We used diets enriched in PUFAs and modeled the dietary FA ratios to those typically consumed or recommended for human consumption. The Soy diet is most similar to the average Western diet $(\omega-6 / \omega-3 \sim 10: 1-30: 1)$, whereas the $\mathrm{M} / \mathrm{S}$ diet is modeled after the NIH recommendations $(\omega-6 / \omega-3$ $\sim 2: 1-1: 1)$. In contrast, the Men diet is highly enriched in $\omega-3$ FAs and is analogous to the intravenous lipid emulsion used to successfully reverse total parenteral nutrition-associated cholestasis (14). In this study, only the Men diet significantly inhibited tumor growth, supporting the idea that the efficacy of $\omega-3$ FAs is closely correlated with the $\omega-6 / \omega-3$ ratio and less so with the total $\omega-3$ content.

The mechanisms by which $\omega-3$ FAs inhibit neuroblastoma growth have been studied in vitro $(15,16)$ but not in vivo. 
Table 2. Percentage of major cis fatty acids and lipid ratios in tumors of mice-fed Control or Soy-, M/S-, or Men-based diets

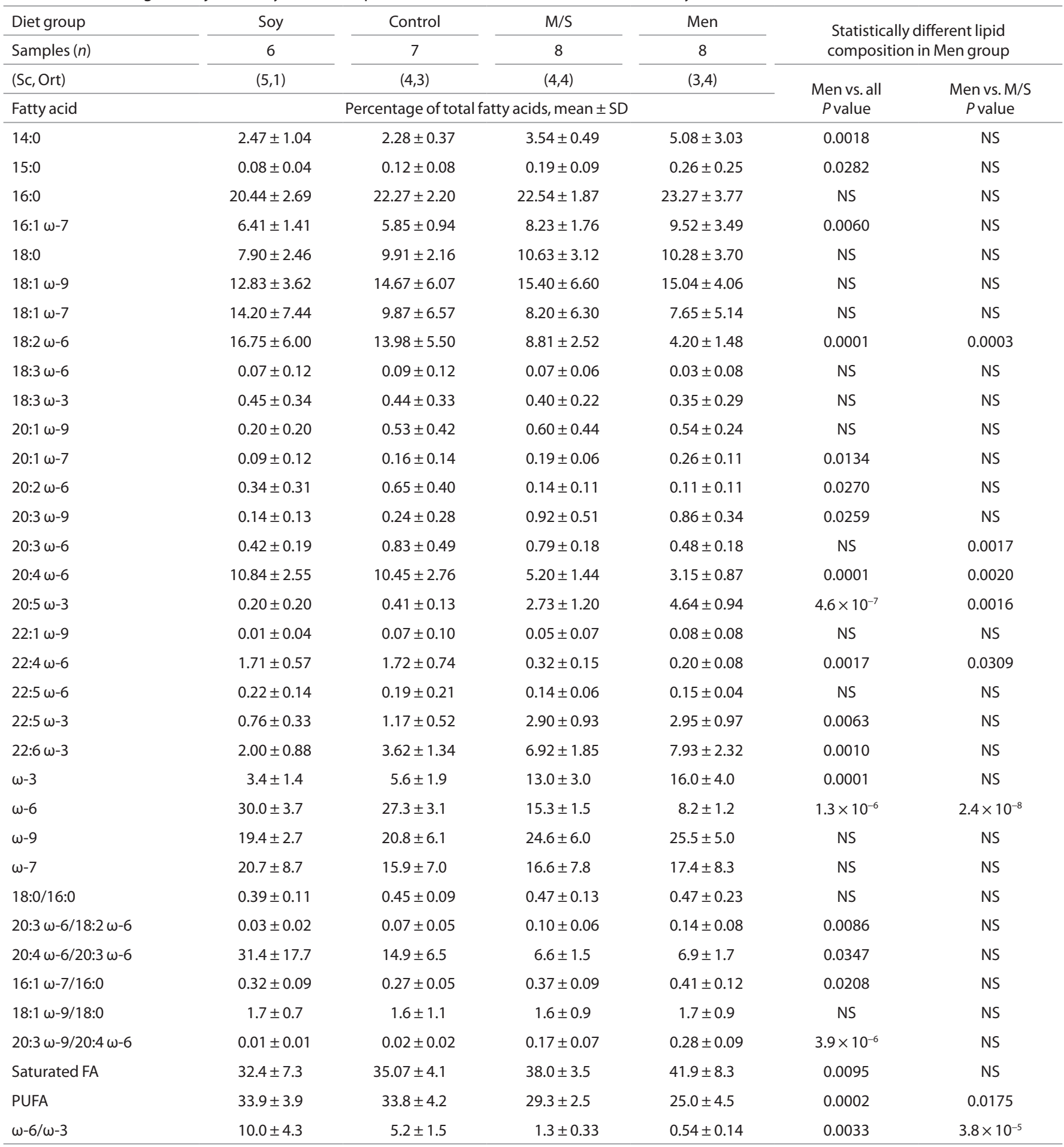

All, Control, Soy, M/S groups combined.; FA, fatty acid; Men, 10\% wt/wt menhaden oil; M/S, 7.5\% wt/wt menhaden oil + 2.5\% wt/wt soybean oil; NS, not significant; Ort, orthotopic tumor; PUFA, polyunsaturated fatty acid; Sc, subcutaneous tumor; Soy, 10\% w/w soybean oil.

In other tumor models, it has been suggested that the in vivo antitumor effects of $\omega-3$ FAs may be mediated by inhibition of tumor cell proliferation and/or induction of apoptosis (17), increased mitochondrial damage through an increase in reactive oxygen species, anti-inflammatory effects, and/or antiangiogenic effects via inhibition of MVD and COX-2 $(17,18)$.
We have performed several studies to gain insight into whether these mechanisms contribute to the inhibition of neuroblastoma tumor growth in vivo.

Our work does not support a direct antitumor or a prodifferentiation effect. Although $\omega-3$ FAs inhibited neuroblastoma cell growth in vitro, no differences in proliferation or apoptosis 
Table 3. Profile of cis fatty acids and lipid ratios in Men-fed vs. M/S-, Soy-, and Control-fed tissues

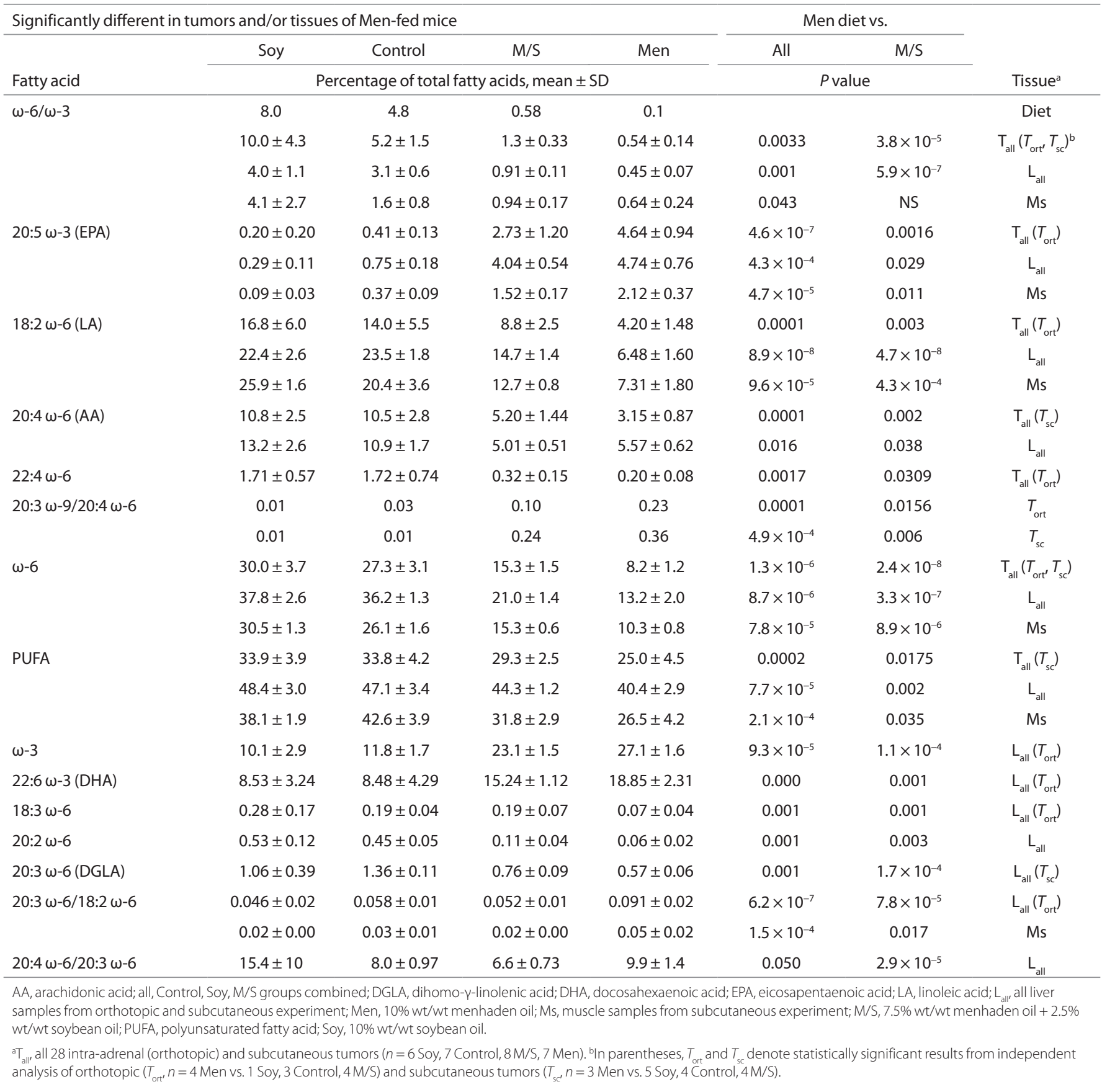

were found in vivo, possibly due to the extensive necrosis associated with this tumor model. Furthermore, while $\omega-6$ and, to a lesser extent, $\omega-3$ and $\omega-9$ FAs are reported to differentiate neuroblastoma cells in vitro $(19,20)$, no differentiation was found in any treatment group, despite significant differences in the $\omega-3$ and $\omega-6$ FA levels. Finally, the Men diet as monotherapy did not reduce the MVD in the tumors or affect COX-2 expression.

We found that Men tumors did display some evidence of increased mitochondrial damage as evidenced by the mitochondrial appearance on electron microscopy and the OxPhos complex IV subunit staining (data not shown). Although these results do not provide definitive evidence of mitochondrial dysfunction, they suggest that this may be a mechanism by which $\omega-3$ FAs mediate their antitumor effects. More in-depth studies will be needed to evaluate these preliminary findings on mitochondrial dysfunction.

This study is the first to demonstrate that $\omega-3$ FA inhibition of tumor growth may be related to a high triene/tetraene ratio within tumors. Neuroblastoma cells have been previously shown to lack $\omega-3$ and $\omega-6$ FAs in vitro $(16,21)$. In our in vivo studies, tumors from animals on the Men diet had an elevated triene/tetraene ratio whereas a normal ratio was maintained in other tissues. Although this does not indicate the development of essential FA deficiency in the intact animal, it does 
Table 4. Percentage of major cis fatty acids and lipid ratios in livers of mice fed Control or Soy-, M/S-, or Men-based diets

\begin{tabular}{|c|c|c|c|c|c|c|}
\hline Diet group & Soy & Control & $\mathrm{M} / \mathrm{S}$ & Men & \multirow{2}{*}{\multicolumn{2}{|c|}{$\begin{array}{l}\text { Statistically different lipid } \\
\text { composition in Men group }\end{array}$}} \\
\hline Samples ( $n)$ & 5 & 6 & 8 & 7 & & \\
\hline (Sc,Ort) & $(4,1)$ & $(5,1)$ & $(4,4)$ & $(5,2)$ & \multirow{2}{*}{$\begin{array}{l}\text { Men vs. All } \\
P \text { value }\end{array}$} & \multirow{2}{*}{$\begin{array}{l}\text { Men vs. M/S } \\
\quad P \text { value }\end{array}$} \\
\hline Fatty acid & \multicolumn{4}{|c|}{ Percentage of total fatty acids, mean \pm SD } & & \\
\hline 14:0 & $0.35 \pm 0.15$ & $0.26 \pm 0.08$ & $0.98 \pm 0.29$ & $1.06 \pm 0.47$ & 0.009 & NS \\
\hline 15:0 & $0.06 \pm 0.0$ & $0.08 \pm 0.02$ & $0.21 \pm 0.06$ & $0.23 \pm 0.06$ & 0.004 & NS \\
\hline $16: 0$ & $21.41 \pm 0.82$ & $23.16 \pm 1.65$ & $24.06 \pm 1.86$ & $25.33 \pm 1.39$ & 0.004 & NS \\
\hline $16: 1 \omega-7$ & $1.53 \pm 1.12$ & $1.32 \pm 0.22$ & $4.11 \pm 1.75$ & $5.25 \pm 2.17$ & 0.002 & NS \\
\hline $18: 0$ & $11.24 \pm 2.96$ & $11.48 \pm 1.59$ & $8.48 \pm 0.98$ & $9.75 \pm 1.48$ & NS & 0.034 \\
\hline $18: 1 \omega-9$ & $9.41 \pm 7.32$ & $13.36 \pm 2.87$ & $15.92 \pm 3.36$ & $15.45 \pm 3.33$ & NS & NS \\
\hline $18: 1 \omega-7$ & $6.65 \pm 7.75$ & $1.04 \pm 2.01$ & $1.17 \pm 1.94$ & $1.88 \pm 3.38$ & NS & NS \\
\hline $18: 2 \omega-6$ & $22.44 \pm 2.64$ & $23.47 \pm 1.83$ & $14.68 \pm 1.40$ & $6.48 \pm 1.60$ & $8.9 \times 10^{-8}$ & $4.7 \times 10^{-8}$ \\
\hline $18: 3 \omega-6$ & $0.28 \pm 0.17$ & $0.19 \pm 0.04$ & $0.19 \pm 0.07$ & $0.07 \pm 0.04$ & 0.001 & 0.001 \\
\hline $18: 3 \omega-3$ & $0.74 \pm 0.64$ & $0.52 \pm 0.12$ & $0.98 \pm 0.27$ & $0.50 \pm 0.17$ & 0.048 & 0.001 \\
\hline $20: 1 \omega-9$ & $0.30 \pm 0.14$ & $0.23 \pm 0.09$ & $0.31 \pm 0.09$ & $0.25 \pm 0.03$ & NS & NS \\
\hline $20: 1 \omega-7$ & $0.19 \pm 0.19$ & $0.13 \pm 0.12$ & $0.08 \pm 0.02$ & $0.07 \pm 0.02$ & NS & NS \\
\hline $20: 2 \omega-6$ & $0.53 \pm 0.12$ & $0.45 \pm 0.05$ & $0.11 \pm 0.04$ & $0.06 \pm 0.02$ & 0.001 & 0.003 \\
\hline $20: 3 \omega-9$ & $0.00 \pm 0.00$ & $0.00 \pm 0.00$ & $0.06 \pm 0.01$ & $0.05 \pm 0.03$ & 0.018 & NS \\
\hline $20: 3 \omega-6$ & $1.06 \pm 0.39$ & $1.36 \pm 0.11$ & $0.76 \pm 0.09$ & $0.57 \pm 0.06$ & 0.001 & $1.7 \times 10^{-4}$ \\
\hline $20: 4 \omega-6$ & $13.21 \pm 2.60$ & $10.92 \pm 1.72$ & $5.01 \pm 0.51$ & $5.57 \pm 0.62$ & 0.016 & 0.038 \\
\hline $20: 5 \omega-3$ & $0.29 \pm 0.11$ & $0.75 \pm 0.18$ & $4.04 \pm 0.54$ & $4.74 \pm 0.76$ & $4.2 \times 10^{-4}$ & 0.029 \\
\hline $22: 1 \omega-9$ & $0.00 \pm 0.00$ & $0.00 \pm 0.00$ & $0.00 \pm 0.00$ & $0.00 \pm 0.00$ & NS & NS \\
\hline $22: 4 \omega-6$ & $0.52 \pm 0.14$ & $0.19 \pm 0.10$ & $0.24 \pm 0.18$ & $0.29 \pm 0.25$ & NS & NS \\
\hline $22: 5 \omega-6$ & $0.32 \pm 0.07$ & $0.08 \pm 0.05$ & $0.13 \pm 0.04$ & $0.18 \pm 0.04$ & NS & 0.013 \\
\hline $22: 5 \omega-3$ & $0.49 \pm 0.18$ & $0.72 \pm 0.09$ & $2.86 \pm 0.35$ & $3.03 \pm 0.41$ & 0.002 & NS \\
\hline $22: 6 \omega-3$ & $8.53 \pm 3.24$ & $8.48 \pm 4.29$ & $15.24 \pm 1.12$ & $18.85 \pm 2.31$ & $3.4 \times 10^{-5}$ & 0.001 \\
\hline$\omega-3$ & $10.1 \pm 2.9$ & $11.8 \pm 1.7$ & $23.1 \pm 1.5$ & $27.1 \pm 1.6$ & $9.3 \times 10^{-5}$ & $1.1 \times 10^{-4}$ \\
\hline$\omega-6$ & $37.8 \pm 2.6$ & $36.2 \pm 1.3$ & $21.0 \pm 1.4$ & $13.2 \pm 2.0$ & $8.7 \times 10^{-6}$ & $3.3 \times 10^{-7}$ \\
\hline$\omega-9$ & $10.9 \pm 8.1$ & $14.7 \pm 2.9$ & $20.1 \pm 3.7$ & $20.8 \pm 4.7$ & 0.037 & NS \\
\hline$\omega-7$ & $8.34 \pm 7.7$ & $2.50 \pm 2.1$ & $5.36 \pm 2.2$ & $7.20 \pm 3.0$ & NS & NS \\
\hline $18: 0 / 16: 0$ & $0.52 \pm 0.13$ & $0.50 \pm 0.09$ & $0.35 \pm 0.05$ & $0.39 \pm 0.08$ & NS & NS \\
\hline $20: 3 \omega-6 / 18: 2 \omega-6$ & $0.046 \pm 0.02$ & $0.058 \pm 0.01$ & $0.052 \pm 0.01$ & $0.091 \pm 0.02$ & $6.2 \times 10^{-7}$ & $7.8 \times 10^{-5}$ \\
\hline $20: 4 \omega-6 / 20: 3 \omega-6$ & $15.4 \pm 10$ & $8.0 \pm 0.97$ & $6.6 \pm 0.73$ & $9.9 \pm 1.4$ & 0.050 & $2.9 \times 10^{-5}$ \\
\hline $16: 1 \omega-7 / 16: 0$ & $0.072 \pm 0.05$ & $0.057 \pm 0.01$ & $0.17 \pm 0.08$ & $0.21 \pm 0.08$ & 0.006 & NS \\
\hline $18: 1 \omega-9 / 18: 0$ & $0.97 \pm 1.0$ & $1.2 \pm 0.4$ & $1.9 \pm 0.56$ & $1.6 \pm 0.46$ & NS & NS \\
\hline $20: 3 \omega-9 / 20: 4 \omega-6$ & 0.000 & 0.000 & $0.011 \pm 0.002$ & $0.009 \pm 0.004$ & 0.040 & NS \\
\hline Saturated FA & $33.5 \pm 3.2$ & $36.8 \pm 3.8$ & $34.1 \pm 1.9$ & $36.7 \pm 1.3$ & 0.009 & 0.005 \\
\hline PUFA & $48.4 \pm 3.0$ & $47.1 \pm 3.4$ & $44.3 \pm 1.2$ & $40.4 \pm 2.9$ & $7.7 \times 10^{-5}$ & 0.002 \\
\hline$\omega-6 / \omega-3$ & $4.0 \pm 1.1$ & $3.1 \pm 0.6$ & $0.91 \pm 0.11$ & $0.45 \pm 0.07$ & 0.001 & $5.9 \times 10^{-7}$ \\
\hline
\end{tabular}

All, Control, Soy, M/S groups combined; FA, fatty acid; Men, 10\% wt/wt menhaden oil; M/S, 7.5\% wt/wt menhaden oil + 2.5\% wt/wt soybean oil; NS, not significant; Ort, orthotropic tumor; PUFA, polyunsaturated fatty acid; Sc, subcutaneous tumor; Soy, 10\% wt/wt soybean oil.

suggest changes in local eicosanoid metabolism, similar to those seen in essential FA deficiency. As these changes include both those in vascular reactivity and tissue growth, they may have important implications for tumor biology.

The reduction in AA levels in Men tumors is likely correlated with the decreased prevalence of $\mathrm{CD}_{4} 5^{+}$cells. During inflammation, PLA-2 releases AA, and the downstream COX and lipoxygenase metabolites act as early mediators of neuronal injury and neurodegeneration in vitro (22) and in vivo (23). Decreased AA correlates with decreased PLA-2 and lipoxygenase-1, both of which are involved in the generation of AA-derived inflammatory metabolites. Many studies have documented the protumorigenic 
Articles | Barneset al.

Table 5. Percentage of major cis fatty acids and lipid ratios in skeletal muscle of mice fed Control or Soy-, M/S-, or Men-based diets

\begin{tabular}{|c|c|c|c|c|c|c|}
\hline Diet group & Soy & Control & $\mathrm{M} / \mathrm{S}$ & Men & \multicolumn{2}{|c|}{ Comparison between diets } \\
\hline Samples $(n)$ & 5 & 5 & 4 & 5 & \multirow{2}{*}{$\begin{array}{l}\text { Men vs. All } \\
P \text { value }\end{array}$} & \multirow{2}{*}{$\begin{array}{c}\text { Men vs. M/S } \\
P \text { value }\end{array}$} \\
\hline Fatty acid & \multicolumn{4}{|c|}{ Percentage of total fatty acids, mean \pm SD } & & \\
\hline 14:0 & $1.70 \pm 0.26$ & $1.50 \pm 0.59$ & $4.28 \pm 0.64$ & $6.17 \pm 3.06$ & 0.001 & NS \\
\hline 15:0 & $0.11 \pm 0.02$ & $0.19 \pm 0.05$ & $0.33 \pm 0.03$ & $0.59 \pm 0.19$ & $1.1 \times 10^{-5}$ & 0.019 \\
\hline $16: 0$ & $18.98 \pm 1.02$ & $21.97 \pm 2.16$ & $23.75 \pm 1.19$ & $24.82 \pm 3.55$ & 0.015 & NS \\
\hline $16: 1 \omega-7$ & $6.35 \pm 2.27$ & $5.09 \pm 1.83$ & $10.92 \pm 2.25$ & $11.34 \pm 4.77$ & 0.021 & NS \\
\hline $18: 0$ & $4.77 \pm 0.72$ & $6.09 \pm 1.33$ & $4.91 \pm 0.79$ & $6.59 \pm 5.62$ & NS & NS \\
\hline $18: 1 \omega-9$ & $26.06 \pm 2.46$ & $18.63 \pm 4.54$ & $19.86 \pm 1.55$ & $19.56 \pm 5.62$ & NS & NS \\
\hline $18: 1 \omega-7$ & $2.45 \pm 0.53$ & $2.94 \pm 0.33$ & $3.18 \pm 0.15$ & $3.00 \pm 0.67$ & NS & NS \\
\hline $18: 2 \omega-6$ & $25.87 \pm 1.59$ & $20.36 \pm 3.61$ & $12.73 \pm 0.81$ & $7.31 \pm 1.80$ & $9.6 \times 10^{-5}$ & $4.3 \times 10^{-4}$ \\
\hline $18: 3 \omega-6$ & $0.07 \pm 0.02$ & $0.03 \pm 0.02$ & $0.08 \pm 0.02$ & $0.13 \pm 0.08$ & 0.004 & NS \\
\hline $18: 3 \omega-3$ & $1.72 \pm 0.41$ & $1.11 \pm 0.51$ & $1.02 \pm 0.12$ & $0.72 \pm 0.39$ & 0.015 & NS \\
\hline $20: 1 \omega-9$ & $0.98 \pm 0.31$ & $0.69 \pm 0.46$ & $0.74 \pm 0.25$ & $0.96 \pm 0.51$ & NS & NS \\
\hline $20: 1 \omega-7$ & $0.04 \pm 0.03$ & $0.02 \pm 0.03$ & $0.06 \pm 0.02$ & $0.11 \pm 0.07$ & 0.004 & NS \\
\hline $20: 2 \omega-6$ & $0.36 \pm 0.07$ & $0.34 \pm 0.05$ & $0.17 \pm 0.01$ & $0.20 \pm 0.12$ & 0.034 & NS \\
\hline $20: 3 \omega-9$ & $0.04 \pm 0.02$ & $0.02 \pm 0.01$ & $0.02 \pm 0.01$ & $0.03 \pm 0.03$ & NS & NS \\
\hline $20: 3 \omega-6$ & $0.50 \pm 0.05$ & $0.53 \pm 0.12$ & $0.29 \pm 0.03$ & $0.35 \pm 0.04$ & NS & 0.033 \\
\hline $20: 4 \omega-6$ & $3.43 \pm 0.79$ & $4.77 \pm 1.96$ & $1.90 \pm 0.41$ & $2.11 \pm 1.09$ & NS & NS \\
\hline $20: 5 \omega-3$ & $0.09 \pm 0.03$ & $0.37 \pm 0.09$ & $1.52 \pm 0.17$ & $2.12 \pm 0.37$ & $4.7 \times 10^{-5}$ & 0.011 \\
\hline $22: 1 \omega-9$ & $0.03 \pm 0.04$ & $0.02 \pm 0.05$ & $0.00 \pm 0.00$ & $0.00 \pm 0.00$ & NS & NS \\
\hline $22: 4 \omega-6$ & $0.26 \pm 0.08$ & $0.18 \pm 0.05$ & $0.07 \pm 0.02$ & $0.08 \pm 0.04$ & 0.017 & NS \\
\hline $22: 5 \omega-6$ & $0.38 \pm 0.06$ & $0.27 \pm 0.09$ & $0.24 \pm 0.05$ & $0.31 \pm 0.06$ & NS & NS \\
\hline $22: 5 \omega-3$ & $0.84 \pm 0.23$ & $1.73 \pm 0.48$ & $2.35 \pm 0.56$ & $2.61 \pm 0.86$ & 0.011 & NS \\
\hline $22: 6 \omega-3$ & $4.63 \pm 2.17$ & $12.8 \pm 5.1$ & $11.3 \pm 2.3$ & $10.5 \pm 4.8$ & NS & NS \\
\hline$\omega-3$ & $7.29 \pm 2.31$ & $16.06 \pm 5.12$ & $16.26 \pm 2.94$ & $16.00 \pm 5.05$ & NS & NS \\
\hline$\omega-6$ & $30.5 \pm 1.3$ & $26.15 \pm 1.58$ & $15.31 \pm 0.55$ & $10.28 \pm 0.84$ & $7.8 \times 10^{-5}$ & $8.9 \times 10^{-6}$ \\
\hline$\omega-9$ & $32.5 \pm 3.2$ & $23.8 \pm 6.4$ & $30.8 \pm 2.6$ & $30.9 \pm 10.1$ & NS & NS \\
\hline$\omega-7$ & $8.85 \pm 2.11$ & $8.05 \pm 1.77$ & $14.15 \pm 2.112$ & $14.4 \pm 4.5$ & 0.016 & NS \\
\hline $18: 0 / 16: 0$ & $0.25 \pm 0.04$ & $0.27 \pm 0.04$ & $0.21 \pm 0.03$ & $0.25 \pm 0.16$ & NS & NS \\
\hline $20: 3 \omega-6 / 18: 2 \omega-6$ & $0.02 \pm 0.00$ & $0.03 \pm 0.01$ & $0.02 \pm 0.00$ & $0.05 \pm 0.02$ & $1.5 \times 10^{-4}$ & 0.017 \\
\hline $20: 4 \omega-6 / 20: 3 \omega-6$ & $6.9 \pm 1.1$ & $8.64 \pm 2.29$ & $6.55 \pm 0.97$ & $6.1 \pm 2.7$ & NS & NS \\
\hline $16: 1 \omega-7 / 16: 0$ & $0.33 \pm 0.12$ & $0.24 \pm 0.11$ & $0.46 \pm 0.12$ & $0.48 \pm 0.22$ & NS & NS \\
\hline $18: 1 \omega-9 / 18: 0$ & $5.6 \pm 1.1$ & $3.31 \pm 1.46$ & $4.14 \pm 0.85$ & $4.4 \pm 2.2$ & NS & NS \\
\hline $20: 3 \omega-9 / 20: 4 \omega-6$ & $0.01 \pm 0.01$ & $0.01 \pm 0.01$ & $0.01 \pm 0.00$ & $0.02 \pm 0.02$ & 0.023 & NS \\
\hline Saturated FA & $25.9 \pm 1.3$ & $30.02 \pm 2.78$ & $33.48 \pm 2.20$ & $38.5 \pm 6.4$ & 0.001 & NS \\
\hline PUFA & $38.1 \pm 1.9$ & $42.6 \pm 3.9$ & $31.8 \pm 2.9$ & $26.5 \pm 4.2$ & $2.1 \times 10^{-4}$ & 0.035 \\
\hline$\omega-6 / \omega-3$ & $4.1 \pm 2.7$ & $1.63 \pm 0.80$ & $0.94 \pm 0.17$ & $0.64 \pm 0.24$ & 0.043 & NS \\
\hline
\end{tabular}

All, Control, Soy, M/S groups combined; FA, fatty acid; Men, $10 \%$ wt/wt menhaden oil; M/S, 7.5\% wt/wt menhaden oil + 2.5\% wt/wt soybean oil; NS, not significant;

PUFA, polyunsaturated fatty acid; Soy, 10\% wt/wt soybean oil.

role that cytosolic PLA-2- $\alpha$ plays in cancer (13). COX-2 catalyzes the oxygenation of AA released from membrane phospholipids by PLA-2 to generate prostaglandin $\mathrm{H}_{2}$ and downstream angiogenic prostanoids. Despite the constitutive COX-2 expression in neuroblastoma cells, these metabolites are likely to be reduced in the Men tumors, given the dramatic reduction in intratumoral AA and decreased PLA-2.
Finally, we show that the antitumor effects of an $\omega-3$ FAenriched diet are enhanced in the presence of sunitinib. When combined with an $\omega-3$ FA-enriched diet, we found a reduction in tumor proliferation and MVD as compared with single agent therapy.

Our results demonstrate that an $\omega$-3 FA-enriched diet reduces neuroblastoma tumor growth in a murine tumor model. This 
a

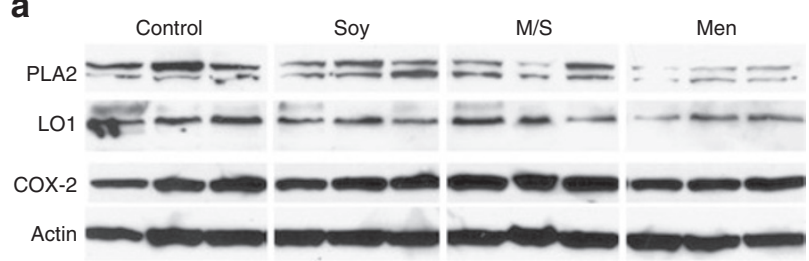

b

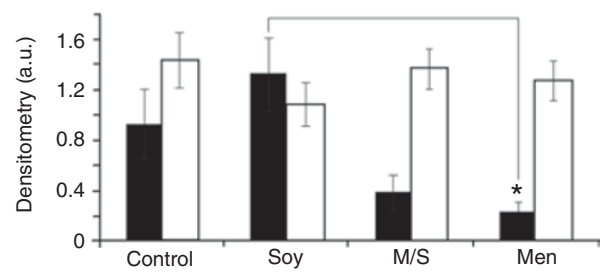

Figure 5. Western blot of PLA-2, LO-1, and COX-2. (a) PLA-2, LO-1, and COX-2 expression in tumors from the different diet groups. Control = actin. (b) Densitometry of normalized PLA-2 (filled bars) and COX-2 (open bars) levels. ${ }^{*} P<0.05$. a.u., arbitrary units; COX, cyclooxygenase; LO-1, lipoxygenase-1; Men, group fed $10 \%$ wt/wt menhaden oil; M/S, group fed $7.5 \%$ wt/wt menhaden oil + $2.5 \%$ wt/wt soybean oil; PLA-2, phospholipase A2.

effect may be the result of changes in local eicosanoid metabolism induced by dietary $\omega-3$ FAs or may be related to a reduction in PLA-2 expression, an altered inflammatory response, or induction of mitochondrial dysfunction. Because prolonged administration of high levels of $\omega-3$ FAs in children is safe $(24,25)$, we propose that $\omega-3$ FAs may be effective in the combination treatment of neuroblastoma.

\section{METHODS}

\section{Cell Lines and Cultures}

SK-NSH cells containing the luciferase transgene (donated by W.A. Weiss and L. Chesler, University of California-San Francisco) were grown in RPMI-1640 containing 10\% heat-inactivated fetal bovine serum (Invitrogen/GIBCO, Grand Island, NY) in 5\% $\mathrm{CO}_{2}$. Bovine capillary endothelial cells were grown, as previously described (26). Endothelial and tumor cell survival were assayed, as previously described (26). In brief, neuroblastoma and bovine capillary endothelial cells were plated in $5 \%$ serum (basal media) and treated $24 \mathrm{~h}$ later with full media containing AA, DHA (kindly provided by Martek, Columbia, MD), sunitinib (Pfizer), or hydrogenated coconut oil at $10 \mathrm{pmol} / \mathrm{l}-100 \mu \mathrm{mol} / \mathrm{l}$; full media alone (positive control); or fresh basal media (negative control). Cell numbers were measured and analyzed, as previously described (26) at the time of challenge and $72 \mathrm{~h}$ after treatment. Lipid bodies were stained and enumerated, as previously described (27). Following these experiments, a second neuroblastoma cell line (IMR-32; ATCC, Manassas, VA) was grown in minimal essential media supplemented with $10 \%$ heat-inactivated fetal bovine serum (Invitrogen/GIBCO) in 5\% CO2 and treated with DHA and AA at $25-100 \mu \mathrm{mol} / \mathrm{l}$, as described above.

\section{Experimental Animals}

Six- to eight-week-old male mice with severe combined immunodeficiency (Massachusetts General Hospital, Boston, MA) were fed ad libitum one of four diets differing in FA content: (i) Control (Prolab Autoclavable 5904; Agway, Syracuse, NY); or AIN-93G (Dyets, Bethlehem, PA) modified with (ii) $10 \% \mathrm{wt} / \mathrm{wt}$ soybean oil (Soy), (iii) $7.5 \% \mathrm{wt} / \mathrm{wt}$ menhaden oil $+2.5 \% \mathrm{wt} / \mathrm{wt}$ soybean oil (M/S), or (iv) $10 \%$ wt/wt menhaden oil (Men) (Table 1). For pretreatment studies, diets were initiated 3 weeks prior to tumor implantation and continued during tumor growth. For treatment studies, mice were randomized by tumor size $\left(>100 \mathrm{~mm}^{3}\right.$ or photon flux $5 \times 10^{6}, \sim 7$ days postimplantation), at which time they were switched to Men diet (Men-2) and gavaged daily with either sunitinib $(20 \mathrm{mg} / \mathrm{kg} /$ day $)$ or vehicle.

\section{Tumor Cell Inoculation and Measurements}

Subcutaneous model: A total of $1.7 \times 10^{6}$ neuroblastoma cells (in $100 \mu \mathrm{l}$ phosphate-buffered saline) were subcutaneously injected into the flank. Tumors were measured by calipers triweekly and tumor volumes calculated (volume $=$ length $\times$ width $^{2} \times 0.52$ ).

Orthotopic model: A 1-cm flank incision was made and $1 \times 10^{5}$ neuroblastoma cells (in $5 \mu \mathrm{l}$ in phosphate-buffered saline) were injected intra-adrenally (28). Tumors were monitored by luciferase imaging biweekly. At killing (16-21 days postimplantation), tumor, liver, and skeletal muscle (rectus femoris) were collected. Tumors were weighed and non-necrotic portions were saved for electron microscopy or histology or frozen at $-80^{\circ} \mathrm{C}$ for lipid/protein analysis. All other tissues were frozen at $-80^{\circ} \mathrm{C}$ for lipid analysis.

\section{Transmission Electron Microscopy}

Immediately after tumor resection, dissected tumor was cut into $1-\mathrm{mm}^{3}$ pieces, fixed, and processed according to an established protocol (29). Ultrathin sections were observed using a Tecnai G2 Spirit electron microscope (FEI Company, Hillsboro, OR) at $120 \mathrm{kV}$.

\section{Immunohistochemistry}

Paraffin-embedded sections were dewaxed, rehydrated, and their endogenous peroxidases inactivated according to standard methods. For antigen retrieval, tumor slides were microwaved (CD45, Ki67) or incubated with proteinase K (TUNEL, CD31; BD Biosciences, Franklin Lakes, NJ). For MVD, rat anti-mouse CD31 (BD Biosciences), biotinylated anti-rat (Vector Laboratories, Burlingame, CA), biotinylated tyramide kit (Perkin Elmer, Waltham, MA), and 3,3'-diaminobenzidine (Dako, Glostrup, Denmark) were used. The most intense CD31stained area plus nine consecutive fields were photographed $(\times 200)$ and analyzed as average MVD (vessels $/ \mathrm{mm}^{2}$ ) or cross-sectional MVD (average vessel number crossed by a horizontal line from 10 $\times 10$ grid). For inflammatory density, slides were stained with rat anti-mouse CD45 (BD Biosciences) and the rat on mouse immunohistochemistry kit (Biogenex, Fremont, CA). $\mathrm{CD}^{+} 5^{+}$cells were manually counted and averaged over 20 fields $(\times 400)$. For proliferation indexes, rabbit anti-Ki67 (Vector Laboratories), biotinylated antirabbit (Vector Laboratories), fluorescein tyramide kit (Perkin Elmer), and 4',6-diamidino-2-phenylindole-containing Vectashield mounting medium (Vector Laboratories) were used. Apoptotic cells were stained using the terminal deoxynucleotidyl transferase (TdT)-mediated 2'-deoxyuridine, $5^{\prime}$-triphosphate in situ nick-end labeling (TUNEL) technique. Ki67- and TUNEL-stained cells were manually counted in $10-15$ images spanning the entire tumor section $(\times 400)$.

\section{Western Blot Analysis}

Tumors were lysed in radioimmunoprecipitation assay buffer with protease inhibitors (Roche, Mannheim, Germany) and run in sodium dodecyl sulfate-polyacrylamide Tris-acetate gels (Invitrogen, Carlsbad, CA), transferred to polyvinylidene fluoride membranes (Invitrogen), and blocked with milk. Membranes were incubated with rabbit antiCOX-2 (Clone SP21) antibody (Lab Vision, Fremont, CA), rabbit antiPLA-2 (Abcam, Cambridge, MA), rabbit anti-lipoxygenase-1 (Santa Cruz Biotechnology, Santa Cruz, CA), or mouse anti-glyceraldehyde 3-phosphate dehydrogenase (Bioscience Research Reagent, Temecula, CA) overnight, followed by their respective secondary horseradish peroxidase-conjugated rabbit or mouse IgG antibodies (GE Healthcare Life Sciences, Piscataway, NJ). Proteins were visualized with the Amersham ECL detection system (Life Sciences, Piscataway, NJ).

\section{FA Analysis}

Total FAs were extracted from the tumor, liver, and muscle tissues per the modified Folch method (30). The FA analysis was performed on a Hewlett-Packard $6890 \mathrm{~N}$ gas chromatograph (GMI, Ramsey, MN) 
coupled to an HP-5975B mass spectrometer equipped with Supelcowax SP-10 capillary column (GMI). FA concentrations (nmol/g tissue) were calculated by proportional comparison of peak areas with the area of the 17:0 internal standard. The percentage composition of FAs and relevant lipid ratios from each tissue type are shown in Tables 2,4 , and 5.

\section{Statistical Analysis}

The results of each independent experiment were normalized to the mean of the control groups and averaged with replicate experiments. Data are reported as treatment over control. Analyses were performed using SigmaStat software (version 3.0, Aspire Software International, Ashburn, VA; http://www.aspiresoftwareintl.com). Differences in the mean values among the treatment groups were analyzed by ANOVA for significance and compared by pairwise multiple-comparison procedures (Holm-Sidak method). Data not normally distributed were analyzed by Kruskal-Wallis ANOVA on ranks, and pairwise comparisons were performed using Dunn's method. $P$ values for FA profiles were calculated using the Student's $t$ test. Samples were standardized to the $z$-score and hierarchical clustering was performed using the opensource clustering software Cluster 3.0 and Java TreeView.

\section{Ethics Statement}

All procedures were in accordance with National Institutes of Health standards and approved by the Boston Children's Hospital Institutional Animal Care and Use Committee.

\section{ACKNOWLEDGMENTS}

We thank Anne Dvorak and Jo-Anne Vergilio for their invaluable assistance in reviewing electron microscopy and histology, Diane Bielenberg and Bruce Bistrian for their valued discussions, and Kristin Johnson for her graphic assistance. This work is dedicated to the memory of Judah Folkman.

\section{STATEMENT OF FINANCIAL SUPPORT}

This work was supported by Saint Baldrick's Foundation (C.M.B., E.A.C.), Circle of Friends (C.M.B., F.C., D.P.), the Children's Hospital Surgical Foundation (E.A.C., H.D.L., D.N., E.M.F., J.F., M.P.), the Vascular Biology Program (C.M.B., D.P., H.D.L, S.S., F.C., D.P., D.C., C.B., D.N., E.M.F., M.K., J.F., M.P.), and the Joshua Ryan Rappaport Fellowship (H.D.L.).

\section{REFERENCES}

1. Maris JM. The biologic basis for neuroblastoma heterogeneity and risk stratification. Curr Opin Pediatr 2005;17:7-13.

2. Berquin IM, Edwards IJ, Chen YQ. Multi-targeted therapy of cancer by omega-3 fatty acids. Cancer Lett 2008;269:363-77.

3. Simopoulos AP. The importance of the omega-6/omega-3 fatty acid ratio in cardiovascular disease and other chronic diseases. Exp Biol Med (Maywood) 2008;233:674-88.

4. Iigo M, Nakagawa T, Ishikawa $\mathrm{C}$, et al. Inhibitory effects of docosahexaenoic acid on colon carcinoma 26 metastasis to the lung. Br J Cancer 1997;75:650-5.

5. Yuri T, Danbara N, Tsujita-Kyutoku M, et al. Dietary docosahexaenoic acid suppresses $\mathrm{N}$-methyl-N-nitrosourea-induced mammary carcinogenesis in rats more effectively than eicosapentaenoic acid. Nutr Cancer 2003;45:211-7.

6. Kelavkar UP, Hutzley J, Dhir R, Kim P, Allen KG, McHugh K. Prostate tumor growth and recurrence can be modulated by the omega-6:omega- 3 ratio in diet: athymic mouse xenograft model simulating radical prostatectomy. Neoplasia 2006;8:112-24.

7. Lim K, Han C, Dai Y, Shen M, Wu T. Omega-3 polyunsaturated fatty acids inhibit hepatocellular carcinoma cell growth through blocking betacatenin and cyclooxygenase-2. Mol Cancer Ther 2009;8:3046-55.

8. Gleissman H, Segerström L, Hamberg M, et al. Omega-3 fatty acid supplementation delays the progression of neuroblastoma in vivo. Int J Cancer 2011;128:1703-11.

9. Shusterman S, Maris JM. Prospects for therapeutic inhibition of neuroblastoma angiogenesis. Cancer Lett 2005;228:171-9.

10. Rupniak HT, Rein G, Powell JF, et al. Characteristics of a new human neuroblastoma cell line which differentiates in response to cyclic adenosine 3':5'-monophosphate. Cancer Res 1984;44:2600-7.
11. Accioly MT, Pacheco P, Maya-Monteiro CM, et al. Lipid bodies are reservoirs of cyclooxygenase- 2 and sites of prostaglandin-E2 synthesis in colon cancer cells. Cancer Res 2008;68:1732-40.

12. Linkous AG, Yazlovitskaya EM, Hallahan DE. Cytosolic phospholipase A2 and lysophospholipids in tumor angiogenesis. J Natl Cancer Inst 2010;102:1398-412.

13. Tosato G, Segarra M, Salvucci O. Cytosolic phospholipase A2 $\{\alpha\}$ and cancer: a role in tumor angiogenesis. J Natl Cancer Inst 2010;102: 1377-9.

14. Gura KM, Duggan CP, Collier SB, et al. Reversal of parenteral nutrition-associated liver disease in two infants with short bowel syndrome using parenteral fish oil: implications for future management. Pediatrics 2006;118:e197-201.

15. Langelier B, Alessandri JM, Perruchot MH, Guesnet P, Lavialle M. Changes of the transcriptional and fatty acid profiles in response to $\mathrm{n}-3$ fatty acids in SH-SY5Y neuroblastoma cells. Lipids 2005;40:719-28.

16. Lindskog M, Gleissman H, Ponthan F, Castro J, Kogner P, Johnsen JI. Neuroblastoma cell death in response to docosahexaenoic acid: sensitization to chemotherapy and arsenic-induced oxidative stress. Int J Cancer 2006;118:2584-93.

17. Calviello G, Di Nicuolo F, Gragnoli S, et al. n-3 PUFAs reduce VEGF expression in human colon cancer cells modulating the COX-2/PGE2 induced ERK-1 and -2 and HIF-1alpha induction pathway. Carcinogenesis 2004;25:2303-10.

18. Olivo SE, Hilakivi-Clarke L. Opposing effects of prepubertal low- and high-fat $\mathrm{n}-3$ polyunsaturated fatty acid diets on rat mammary tumorigenesis. Carcinogenesis 2005;26:1563-72.

19. Burdge GC, Rodway H, Kohler JA, Lillycrop KA. Effect of fatty acid supplementation on growth and differentiation of human IMR-32 neuroblastoma cells in vitro. J Cell Biochem 2000;80:266-73.

20. Di Loreto S, D’Angelo B, D’Amico MA, et al. PPARbeta agonists trigger neuronal differentiation in the human neuroblastoma cell line SH-SY5Y. J Cell Physiol 2007;211:837-47.

21. Reynolds LM, Dalton CF, Reynolds GP. Phospholipid fatty acids and neurotoxicity in human neuroblastoma SH-SY5Y cells. Neurosci Lett 2001;309:193-6.

22. Smalheiser NR, Dissanayake S, Kapil A. Rapid regulation of neurite outgrowth and retraction by phospholipase A2-derived arachidonic acid and its metabolites. Brain Res 1996;721:39-48.

23. Montine TJ, Markesbery WR, Zackert W, Sanchez SC, Roberts LJ $>2^{\text {nd, }}$, Morrow JD. The magnitude of brain lipid peroxidation correlates with the extent of degeneration but not with density of neuritic plaques or neurofibrillary tangles or with APOE genotype in Alzheimer's disease patients. Am J Pathol 1999;155:863-8.

24. Gura KM, Lee S, Valim C, et al. Safety and efficacy of a fish-oil-based fat emulsion in the treatment of parenteral nutrition-associated liver disease. Pediatrics 2008;121:e678-86.

25. Le HD, Meisel JA, de Meijer VE, Gura KM, Puder M. The essentiality of arachidonic acid and docosahexaenoic acid. Prostaglandins Leukot Essent Fatty Acids 2009;81:165-70.

26. Mukhopadhyay S, Barnés CM, Haskel A, Short SM, Barnes KR, Lippard SJ. Conjugated platinum(IV)-peptide complexes for targeting angiogenic tumor vasculature. Bioconjug Chem 2008;19:39-49.

27. Bozza PT, Payne JL, Morham SG, Langenbach R, Smithies O, Weller PF. Leukocyte lipid body formation and eicosanoid generation: cyclooxygenase-independent inhibition by aspirin. Proc Natl Acad Sci USA 1996;93:11091-6.

28. Salcedo R, Stauffer JK, Lincoln E, et al. IL-27 mediates complete regression of orthotopic primary and metastatic murine neuroblastoma tumors: role for CD8+ T cells. J Immunol 2004;173:7170-82.

29. Dvorak AM, Monohan-Earley RA. Diagnostic Ultrastructural Pathology. I. A Text-Atlas of Case Studies Illustrating the Correlative Clinical-Ultrastructural Pathologic Approach to Diagnosis. CRC Press: Boca Raton, FL, 1992:127-136.

30. Folch J, Lees M, Sloane Stanley GH. A simple method for the isolation and purification of total lipides from animal tissues. J Biol Chem 1957;226:497-509. 